\section{Diverse Pathophysiology of Sudden Unexpected Death in Epilepsy in Children}

We read with interest the review article by Garg and Sharma [1] on sudden unexplained death in epilepsy (SUDEP) in the pediatric population. We have the following comments.

A pathophysiological mechanism of SUDEP not considered by the authors is Takotsubo syndrome, also known as stunned myocardium or broken heart syndrome. Takotusbo syndrome is an acute onset, usually reversible cardiomyopathy, mainly of the left ventricle, morphologically and functionally characterized by focal or global dyskinesia, hypokinesia, or akinesia of the left ventricular myocardium, resulting in low output failure [2]. Though the outcome is usually fair, it can be fatal in isolated cases, particularly in those with the global type. The syndrome is triggered by physical or emotional stress, associated with a massive dumping of catecholamines (catecholamine storm). It is considered that the sudden overstimulation of adrenergic receptors on the surface of cardiomyocytes results in contractile dysfunction and thus acute heart failure [2]. Epilepsy is the most frequent central nervous system trigger of Takotsubo syndrome [2]. Since it can be complicated by ventricular arrhythmias [2], patients experiencing Takotsubo syndrome may not only die suddenly from acute heart failure but also from asystole or ventricular fibrillation [2].

A second pathophysiological mechanism not considered is neurogenic pulmonary edema (NPE) [3]. NPE is characterized by acutely developing pulmonary edema within minutes or hours following an acute lesion of the central nervous system [3], which usually resolves spontaneously within 24-48 hours after onset [4]. Central nervous system triggers of NPE so far reported include enterovirus 71-associated brainstem encephalitis, subarachnoid bleeding, intracerebral bleeding, traumatic brain injury, stroke, hypoxia, hydrocephalus, or epilepsy, usually with generalized tonic-clonic seizures [3]. NPE may occur after a single seizure or multiple seizures. In a retrospective study of 47 patients, NPE was found on computed tomography scans of the lungs in $19 \%$ of the patients experiencing a generalized tonic clonic seizure [5].

Overall, patients with epilepsy, particularly those with poor seizure control, polytherapy with anti-seizure drugs, poor compliance, and multiple comorbidities, should be prospectively screened for cardiac and pulmonary disease by electrocardiographic monitoring, echocardiography, stress tests, and pulmonary function tests. Epilepsy patients at risk of cardiac or pulmonary disease should receive primary prophylactic treatment to lower the risk of SUDEP.

Josef Finsterer, ${ }^{1 *}$ AND Fulvio A Scorza ${ }^{2}$

${ }^{1}$ Klinik Landstrasse, Messerli Institute, and ${ }^{2}$ Universidade de Sao Paulo, Austria, Brazil. *fifigs1@yahoo.de

\section{REFERENCES}

1. Garg D, Sharma S. Sudden unexpected death in epilepsy (SUDEP) - What pediatricians need to know. Indian Pediatr. 2020;S097475591600192 [published online ahead of print, 2020 Jun 12].

2. Finsterer J, Wahbi K. CNS disease triggering Takotsubo stress cardiomyopathy. Int J Cardiol. 2014;177:322-29.

3. Finsterer J. Neurological perspectives of neurogenic pulmonary edema. Eur Neurol. 2019;81:94-102.

4. Takagi Y, Imamura T, Endo S, Hayashi K, Akiyama S, Ikuta Y. et al. Neurogenic pulmonary edema following febrile status epilepticus in a 22-month-old infant with multiple respiratory virus co-detection: A case report. BMC Infect Dis. 2020;20:388.

5. Mahdavi Y, Surges R, Nikoubashman O, Dague KO, Brokmann JC, Willmes K, et al. Neurogenic pulmonary edema following seizures: A retrospective computed tomography study. Epilepsy Behav. 2019;94:112-17.

\section{AUTHORS' REPLY}

We thank the reader for their interest in our article [1], and for addressing additional putative pathophysiological mechanisms that may contribute to Sudden unexpected death in epilepsy (SUDEP). The authors suggest a potential role of Takotsubo syndrome. Although it has been well recognised that seizures may trigger this syndrome in adults, the role of this entity in SUDEP in general continues to be debated and in pediatric SUDEP, is definitely uncertain. In a review including 74 patients who developed Takotsubo syndrome in association with a seizure, the age range was $18-82$ years [2]. Of these, a fatal outcome occurred in only two (3\%) patients. This is similar to mortality reported in the International Takotsubo registry [3]. Considering the rarity of fatality, in association with the aforementioned age range, Takotsubo syndrome seems an unlikely contributor to SUDEP pathogenesis in children. Autopsy studies in SUDEP patients indicate that cardiac pathology comprises interstitial fibrosis, myocyte hyper-trophy as well as vascular wall thickening [4]. However, whether these are the effects of multifactorial influences such as anti-seizure medications or even epilepsy itself, or the cause of SUDEP remains unclear. Moreover, none of these features are pathognomonic of "active catecholamine myocarditis" pathology observed in TTS [5].

The authors also suggest a role of neurogenic pulmonary edema (NPE) in the pathogenesis of SUDEP. NPE has been consistently noted in patients with epilepsy and serves almost as a pathological biomarker for SUDEP. However, the reported degree of pulmonary edema has only been to a mild extent, as observed on autopsies in the MORTEMUS study [6]. Additionally, NPE following a seizure tends to be short-lived. Hence, both ante-mortem and post-mortem evidence suggest that NPE following seizures is a common but mild finding, making the link between SUDEP and NPE as a causative factor tenuous.

We agree with the authors' suggestion that underlying cardiac and pulmonary diseases in persons with epilepsy, particularly among those who are refractory to medical 
therapy, should be treated. However, whether this strategy generates a reduction in SUDEP occurrence necessitates more prospec-tively collected data, particularly among children and adolescents.

\section{Divyani Garg ${ }^{1}$ AND Suvasini Sharma ${ }^{2 *}$ Departments of ${ }^{1}$ Neurology and ${ }^{2}$ Pediatrics, Lady Hardinge Medical College, New Delhi, India. *sharma.suvasini@gmail.com}

\section{REFERENCES}

1. Garg D, Sharma S. Sudden Unexpected Death in Epilepsy (SUDEP) - What pediatricians need to know [published online ahead of print, 2020 Jun 12]. Indian Pediatr. 2020;S097475591600192.

2. Finsterer J, Bersano A. Seizure-triggered Takotsubo syndrome rarely causes SUDEP. Seizure. 2015;31:84-7.
3. Templin C, Ghadri JR, Diekmann J, Napp LC, Bataiosu DR, Jaguszewski M, et al. Clinical features and outcomes of Takotsubo (Stress) cardiomyopathy. NEJM. 2015;373:929-38

4. Nascimento FA, Tseng ZH, Palmiere C, Maleszewski JJ, Shiomi T, McCrillis A, et al. Pulmonary and cardiac pathology in sudden unexpected death in epilepsy (SUDEP). Epilepsy Behav. 2017;73:119-25.

5. Mitchell A, Marquis F. Can Takotsubo cardiomyopathy be diagnosed by autopsy? Report of a presumed case presenting as cardiac rupture. BMC Clin Pathol. 2017;17:4.

6. Ryvlin P, Nashef L, Lhatoo SD, Bateman LM, Bird J, Bleasel $\mathrm{A}$, et al. Incidence and mechanisms of cardiorespiratory arrests in epilepsy monitoring units (MORTEMUS): A retrospective study. Lancet Neurol. 2013;12:966-77.

\section{Telephonic Triage and Telemedicine During the Peak of COVID-19 Pandemic - Restricting Exposure to Healthcare Professionals}

We read with interest the article by Mahajan, et al. [1] on the use of telemedicine during the severe acute respiratory syndrome coronavirus 2 (SARS-CoV-2) pandemic. The authors have well summarized the pros and cons of tele-health service. We would like to share our experience with telemedicine used with forward triaging that helps mitigate some of its major limitations and protects healthcare workers (HCWs) from potential exposure.

The guidelines for telemedicine have been eased to enable continued care of non-COVID illnesses [2]. However, it is the chronic illnesses that require holistic care by an entire team,which have taken a backseat in the current scenario. Telehealth needs to be part of routine practice, and not just during emergencies [3]. This focuses on creating a more sustainable model of care, and a telehealth-ready workforce, incorporating telemedicine training even in the medical curriculum [3]. While many countries are using telemedicine to triage COVID suspects, we planned to develop it into a system of care even in the post-pandemic phase $[4,5]$.

Following our initial experience with use of telephonic consults, a new platform that incorporated telemedicine into the existing Hospital Information system (HIS) was launched on 8 June, 2020. A teleconsultation was provided as per schedule, once a telemedicine appointment was taken by the patient, using a simple feature phone, any video calls or images are shared through WhatsApp (Business). Referral to other specialties was also possible to ensure comprehensive care. The prescription was sent to the patient as a PDF document. After a telemedicine consultation, in case the physician felt the need of an in-person visit, the same is again indicated in the online system and patient is allowed physical entry into the OPD after screening on the appointed date and time.

In our hospital, telephonic-only consults were provided to 2477 patients over a period of 45 days (21 April to 7 June, 2020 ) while the new system has enabled provision of care to 10,625 patients over the same time span (8 June to 16 July, 2020) (Fig. 1). Physical consultations constituted only $29 \%$ of the consultations in this period. This also reflects the proportionate reduction in exposure of healthcare staff to potential SARS-CoV-2 carriers.

The system mitigated the limitation of telemedicine by

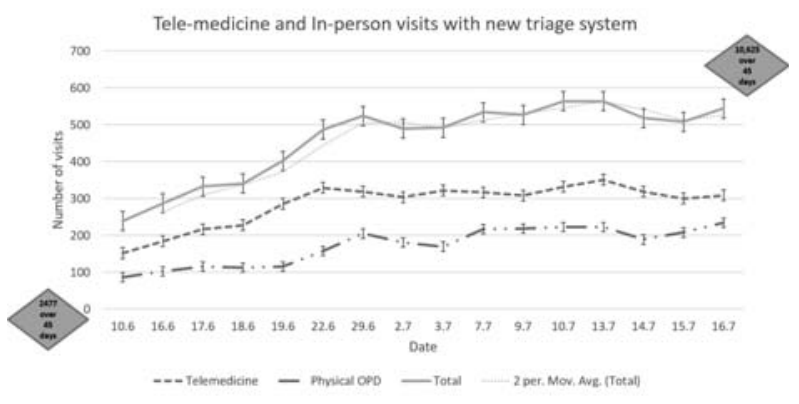

Fig. 1 Line diagram showing the number of patients seen through new OPD system. The red line indicates the number of patients provided in-person visit after telemedicine triage. The green diamonds shows the number of patients cared for- 2477 patients were provided tele-consult before the launch of new platform over 45 days which increased to more than 10000 patients in next 45 days. 